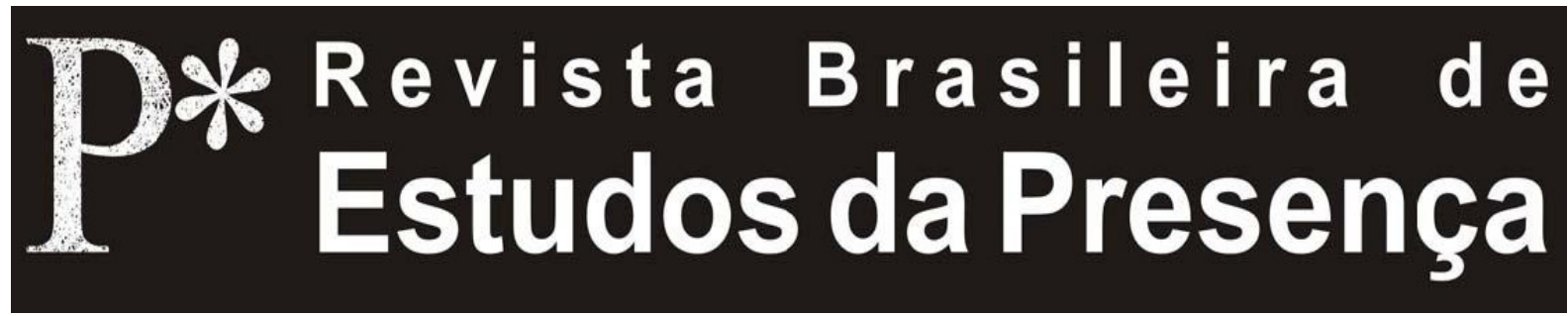

DOI - http://dx.doi.org/10.1590/2237-266026244

ISSN 2237-2660

\title{
A Influência do Trabalho de Delsarte nos Estados Unidos a partir do Final do Século XIX
}

\author{
Nancy Lee Chalfa Ruyter \\ University of California - Irvine, EUA
}

RESUMO - A Influência do Trabalho de Delsarte nos Estados Unidos a partir do Final do Século XIX - Este texto trata da influência de François Delsarte em várias correntes do treinamento corporal, teatral e da preparação vocal nos Estados Unidos, a partir do final do século XIX. Apresenta-se a forma como suas ideias foram desenvolvidas e transformadas no país, desde 1870, tornando-se, em sua última fase, iniciada na década de 1880, uma teoria aplicada a todos os aspectos da vida independentemente de sua corrente europeia. São mencionadas as contribuições de Steele Mackaye, Genevieve Stebbins e Henrietta Hovey, entre outros. Palavras-chave: François Delsarte. Dança. Teatro. Delsartismo Americano. Corpo.

ABSTRACT - The Influence of Delsarte's Work in the United States: Late $19^{\text {th }}$ Century and Beyond - This paper discusses the influence of François Delsarte on bodily motion, theater, and elocution in the United States from the late nineteenth century. It presents his main ideas and how they were developed and transformed in the country since 1870, becoming, in their last phase, which began in the $1880 \mathrm{~s}$, a theory that was applied to all aspects of life, independent from its European form. The contributions of Steele Mackaye, Genevieve Stebbins, Henrietta Hovey and others are mentioned.

Keywords: François Delsarte. Dance. Theater. American Delsartism. Body.

RÉSUMÉ - L'Influence du Travail de Delsarte aux Etats-Unis à partir de la Fin du XI$\mathbf{X}^{\text {ème }}$ Siècle - Ce texte se penche sur l'influence du travail de François Delsartre dans diverses méthodes d'entrainement corporel, théâtral et vocal aux Etats-Unis à partir de la fin du XIX ${ }^{\text {ème }}$ siècle. Il présente la manière dont ses idées ont été développées et transformées dans le pays depuis 1870, pour devenir, dans une dernière phase initiée dans les années 1880, une théorie appliquée à tous les aspects de la vie, indépendement du courant européen. Pour ce faire, il s'appuie sur les contributions de Steele Mackaye, Geneviève Stebbins et Henrietta Hovey, entre autres.

Mots-clés: François Delsarte. Danse. Théâtre. Delsartisme Américain. Corps. 
Neste artigo, apresentarei os norte-americanos que foram importantes na história dos estudos e práticas delsarteanas nos Estados Unidos, a fim de discutir sua atividade de divulgação e adaptação das teorias e práticas de Delsarte nas áreas de atuação, oratória, cultura física feminina, expressão e dança - no desenvolvimento daquilo a que chamo de delsartismo americano. Concentrar-me-ei nas contribuições de Steele Mackaye (1842-94), Genevieve Stebbins (1857-1933 ou após) e Henrietta Hovey (1849-1918); também farei referências a Lewis B. Monroe (1825-79) e William R. Alger (1822-1905). A maior parte deste artigo é inspirada no meu livro The Cultivation of Body and Mind in Nineteenth-Century American Delsartism (1999).

O contexto para a introdução do delsartismo nos Estados Unidos era o campo de elocução (treinamento de voz e dicção para o exercício da fala em público), que vinha se desenvolvendo em escala nacional desde a década de $1820^{1}$. No final do século XIX havia escolas, locais para apresentações, publicações e uma rede nacional de associações profissionais dedicadas a esse campo de atuação. Cada vez mais, a partir de meados do século, alguns instrutores de dicção começaram a enfatizar o gesto e o movimento corporal, e o termo expressão entrou em voga em trabalhos que envolviam cultura física, pantomima, atuação e comunicação interpessoal, bem como o treinamento para falar em público. Enquanto a expressão era ensinada por meio de vários métodos nos Estados Unidos, o que ficou mais conhecido, e em última análise de uso mais amplo, foi o sistema Delsarte Americano (American Delsarte). Esse sistema era formado pela teoria de Delsarte, exercícios práticos e fórmulas de expressão de Mackaye (o que podia ser chamado de sistema Delsarte-Mackaye), exercícios de treinamento físico de origens variadas, e gêneros de performance popular como estátua-viva, pantomima e dança ou formas semelhantes que incorporavam ou refletiam a teoria delsarteana. No final do século XIX, o delsartismo americano havia se espalhado pelos Estados Unidos, envolvendo milhares de estudantes - em sua maior parte mulheres e meninas.

O delsartismo americano passou por três fases durante o seu desenvolvimento. A primeira começou no início da 
década de 1870 e estava intimamente ligada ao treinamento profissional de oradores e atores. A segunda, que emergiu na década de 1880, enfatizava a cultura física para o público em geral. Isso se tornou popular principalmente entre mulheres das classes média e alta. Em seu terceiro aspecto, que também começou na década de 1880 e foi o mais amplo, a teoria estética delsarteana era usada em todos os aspectos da vida. Muitas vezes um único professor oferecia treinamento em dois ou três desses aspectos do treinamento, embora alguns se especializassem em apenas um.

A primeira fase começou com o único aluno americano que se conhece de Delsarte, o famoso ator, dramaturgo, diretor e inovador teatral, James Morrison Steele Mackaye². Mackaye iniciou seu trabalho com Delsarte em Paris, em outubro de 1869. Ele não chegou como um novato inexperiente, pois já desenvolvia abordagens próprias para a expressão, pantomima, ginástica e estética desde a década de 1860 . Delsarte logo descobriu que Mackaye detinha conhecimentos e métodos pedagógicos importantes que poderiam complementar e aprimorar seu próprio trabalho; por essa razão, Delsarte convidou o novo aluno a lecionar com ele. Mackaye continuou sendo estudante de Delsarte, mas em certa medida era também um colega.

Cerca de 20 anos mais tarde, Mackaye pediu à sua esposa, Mary Medberry Mackaye, que escrevesse e publicasse um artigo sobre o trabalho Delsarte-Mackaye. Na sua correspondência não datada sobre o que ela deveria escrever, Mackaye afirmou que havia sido capaz de dominar a série de gestos de Delsarte com muito mais rapidez do que outros estudantes por causa de seu "estudo diligente [...] seguindo um sistema que [ele] mesmo, [Mackaye, teria inventado]". Ele continua:

[D]epois de analisar os movimentos, através de estudos minuciosos descobri os obstáculos físicos da minha própria organização até a compreensão desses movimentos nas minhas próprias ações. Antes de me afastar de Delsarte, eu já havia construído os alicerces de todo o meu sistema e filosofia da ginástica psicológica dos quais resulta a Ginástica Harmônica (Carta sem data para M. M. Mackaye, Delsarte Archive, Luisiana State University, Box 14, Folder 156. O artigo resultante, "Steele Mackaye and François Delsarte", escrito pela Sra. Mary Medberry Steele Mackaye, apareceu na Werner's Voice Magazine, v. 14, jul. 1892, p. 189). 
Se a "Ginástica Harmônica" havia sido desenvolvida por Mackaye ou pelo próprio Delsarte seria um tópico de discussão. Isso foi, de fato, objeto de disputas a partir do início da década de 1870 até o final do século XIX.

A eclosão da Guerra Franco-Prussiana obrigou Mackaye a deixar a França em julho de 1870. Quando retornou aos Estados Unidos, ele foi procurado por dois homens famosos de Boston que haviam ouvido falar sobre o trabalho de Delsarte por intermédio de uma reportagem no Atlantic Monthly (maio de 1871). Ela havia sido escrita pelo jornalista americano Francis A. Durivage, que havia participado de um curso Delsarte-Mackaye em Paris. Os dois indivíduos que entraram em contato com Mackaye eram Lewis B. Monroe - fundador e diretor da Boston University School of Oratory, que na época estudava com Mackaye e convidou-o a dar palestras na escola - e o Reverendo William R. Alger, um clérigo unitarista que começou a estudar com Mackaye e depois continuou seus estudos com o filho de Delsarte, Gustave, em Paris. Os três amigos tentaram levar Delsarte para os Estados Unidos, mas sua morte no verão de 1871 pôs fim a seus planos.

Mackaye dirigiu uma série de escolas nas quais o sistema e seu desenvolvimento eram partes integrantes do programa. Monroe incluiu o sistema de Delsarte no currículo da Boston University School of Oratory e ele próprio o ensinou, também convidando Mackaye como palestrante. Alger também ensinava, escrevia e palestrava sobre Delsarte. Esses três primeiros delsarteanos americanos ensinaram muitos outros, que, por sua vez, ensinaram outros, divulgando os conhecimentos sobre o sistema. Entretanto, dos três, Mackaye foi o único que provavelmente contribuiu significativamente para o desenvolvimento e expansão do conjunto de técnicas delsartianas, como ficou conhecido e praticado nos Estados Unidos - a ginástica harmônica (ou estética) e o vocabulário a que ele chamou de gamas de expressão [gamuts of expression]. Uma demonstração deste último foi muito elogiada em uma de suas palestras em Boston (21 de março de 1871) por um crítico que descreveu as mudanças emocionais de Mackaye como: 
[...] passando pela satisfação, prazer, ternura e amor, para a adoração, e [...] pela aversão, repulsa, inveja e ódio até a fúria [...] [e então] transições do repouso para a jovialidade, tolice e prostração, até a total embriaguez; [...] passando por todas os níveis de distúrbios mentais até a loucura, descendo até o porão da debilidade mental e do idiotismo (Boston Transcript e Boston Advertiser 22 mar. 1871, citado em Percy Mackaye 1, p. 151-52).

A ginástica harmônica e as gamas de expressão tornaram-se elementos fundamentais do delsartismo americano; e é provável que tenha sido Mackaye quem os tenha criado e desenvolvido, mas essa é uma questão ainda sem resposta. Mackaye escreveu alguns artigos, mas nenhum grande tratado sobre o delsartismo tal como ele o ensinava. Ainda assim, as notas feitas para suas palestras e lições estão disponíveis nos arquivos.

Como o próprio Delsarte, Mackaye nunca foi rígido em sua teoria e prática. Ele pesquisava e expandia seu conhecimento e abordagem continuamente. Ele construiu sobre aquilo que havia aprendido com Delsarte, mas também incorporou seu próprio pensamento e inovações ao que ensinava. Ele fundou algumas das primeiras escolas para atores nos Estados Unidos e a sua teoria e metodologia influenciaram uma geração de professores e profissionais de teatro, oratória e cultura física.

A figura representativa da segunda fase do delsartismo americano, no qual a cultura física para o público em geral foi enfatizada, foi Genevieve Stebbins, que acabou se tornando uma das mais importantes divulgadoras do delsartismo americano por intermédio de suas aulas, escritos e performances ${ }^{3}$. Foi ela quem inicialmente desenvolveu o sistema ainda mais o levando para a dança; ela mesma dançava, fazia pantomimas e estátua-viva nas populares matinês para senhoras. Stebbins, assim como muitos dos pioneiros da dança moderna que surgiriam mais tarde, era uma assídua leitora, pesquisadora e escritora - bem como uma artista e professora admirada - e suas publicações deram-lhe visibilidade e estatura no domínio da cultura física e expressão.

$\mathrm{O}$ interesse de Stebbins pela performance e expressão começou a se desenvolver no início de sua vida, como é 
evidenciado pelas performances com pantomimas, canções, danças e estátua-viva que ela fazia quando ainda era criança. Em 1875, então com 18 anos, ela foi para Nova York para seguir a carreira de atriz e sua estreia profissional ocorreu dois anos mais tarde. Ela começou a trabalhar com Mackaye, ou seu assistente, em 1876 ou 1877. Por sugestão dele, ela parou de atuar por dois anos para se dedicar exclusivamente aos estudos com ele, participando em uma de suas produções em 1879, em seguida retornando à sua carreira de atriz. Ela foi tão bem sucedida com o sistema Delsarte-Mackaye que Mackaye lhe pedia para demonstrá-lo nas suas palestras e, às vezes, até mesmo lecionar em seu lugar. Seu relacionamento foi inicialmente caracterizado por cordialidade e respeito mútuo, mas, no fim, se deteriorou à medida que eles se tornaram concorrentes no mundo do delsartismo americano.

A partir de meados da década de 1880 até o início da década de 1890, Stebbins e Mary S. Thompson, sua colega da Boston University School of Oratory, lecionaram na cidade de Nova York e realizaram uma Delsarte Matinee uma vez ao ano em algum grande teatro da cidade. Em 1893, Stebbins e seu marido, Norman Astley, abriram a New York School of Expression, que em 1894 foi incorporada à $F$. Townsend Southwick's School of Oratory. A escola continuou em operação até que Stebbins se aposentou em 1907. Nessa época, Stebbins continuou ativa como palestrante, professora e performer em várias cidades dos Estados Unidos. Ela continuou a se apresentar pelo menos até 1903.

A elaboração de Stebbins do sistema Delsarte teve várias origens ${ }^{4}$. Ela foi inicialmente fundamentada no material teórico e prático que ela aprendeu com Mackaye. Então, em 1881, ela viajou para a Europa com o fim de pesquisar para um livro sobre o sistema Delsarte, lá conhecendo e conversado com Abbé Delaumosne, como também adquirido alguns manuscritos inéditos do próprio Delsarte. Sua primeira publicação, The Delsarte System of Expression foi publicada em seis edições entre 1885 e 1902. A última apresentava várias novas seções e foi reimpressa pela Dance Horizons, em 1977. Ao considerar suas publicações desde a primeira edição de Delsarte System of Expression, passando pelo Genevieve Stebbins System of 
Physical Training de 1913, é evidente que enquanto o trabalho de Delsarte continuou sendo um elemento central para ela, com o passar do tempo ela também incorporou a teoria e a prática de muitas outras fontes. Essas incluíam os trabalhos de outros especialistas em atuação, ginástica, exercícios terapêuticos e antropologia. Além disso, em seus escritos podem-se encontrar referências à literatura, arte, música, religião, filosofia etc. - e, também, à ginástica medicinal sueca e técnicas de respiração da ioga.

A terceira fase do delsartismo americano foi a mais ampla de todas. Seu ideal fundamental era tratar a totalidade da vida como arte e aprimorá-la de acordo com os princípios da estética delsarteana. Essa fase é melhor representada por Henrietta Hovey ${ }^{5}$. Nascida como Henrietta Knapp, ao longo de três casamentos seu sobrenome mudou para Crane, Russel e, finalmente, Hovey (como vou me referir a ela aqui). Hovey lecionou principalmente nos círculos da sociedade até o final de sua vida quando, então, se estabeleceu na região de Los Angeles, entrando em contato com Ted Shawn e Ruth St. Denis, e lecionando na Denishawn School.

Hovey foi a primeira americana a popularizar o sistema Delsarte fora das áreas de atuação e oratória. Com pouco mais de vinte anos de idade, Hovey dava palestras sobre a reforma do vestuário [dress reform] e, em algum momento na década de 1870, com o objetivo de desenvolver sua dicção para apresentações em público, ela se inscreveu na Boston University School of Oratory. Ela aprendeu sobre o sistema Delsarte nas aulas com seu diretor, Lewis B. Monroe, e nas palestras de Mackaye. No final dos anos de 1870 (provavelmente 1878), ela viajou para Paris onde estudou com o filho de Delsarte, Gustave, antes de sua morte em 1879. Ela retornou para os Estados Unidos e iniciou uma carreira de professora que se estenderia por quase quarenta anos. Ela era reconhecida por alguns contemporâneos como uma autoridade em Delsarte mas também rejeitada por outros como uma fraude. A maior parte de suas aparições na imprensa (mais frequentemente nas páginas de coluna social) a descrevia como uma criatura bela e exótica, que atraía senhoras elegantes da classe alta. Elas tinham tempo e recursos para se dedicar às aulas de cultura 
física e expressão deste ícone do delsartismo americano; e, é claro, esses compromissos ofereciam a Hovey renda e prestígio. É difícil chegar a alguma conclusão sobre o ensino de Hovey e a seriedade de seu comprometimento com o trabalho de Delsarte, até chegarmos a seu contato com Ted Shawn. Shawn (1891-1972), com sua parceira Ruth St. Denis (18791968), foi um dos principais pioneiros do que foi desenvolvido no século XX como dança moderna. Hovey certamente impressionou muito a Shawn, e ele aprendeu muito com ela e a respeitava totalmente. Embora ela - assim como Delsarte e Mackaye - tenha planejado escrever vários volumes sobre o sistema Delsarte, ela publicou apenas Yawning (1891), um humilde volume que seria a primeira parte da série (e que foi objeto de comentários zombeteiros na imprensa). Há um longo esboço para outros volumes propostos no Ted Shawn Archive da New York Public Library for the Performing Arts, que parece pressagiar uma obra substancial que, no entanto, nunca foi realizada. O fato de que ele existe nos dá, contudo, uma ideia da abrangência e profundidade do entendimento de Hovey com relação aos princípios delsarteanos.

No final do século XIX, pode-se encontrar o adjetivo delsarteano usado para se referir a professores, escolas, performances e publicações - e até a espartilhos menos restritivos e prejudiciais do que aqueles que as mulheres na moda estavam vestindo. À medida que o delsartismo se espalhava pelos Estados Unidos, ele começou a envolver centenas de professores e milhares de alunos - em sua maioria mulheres e meninas - e, no início do século XX, a versão norte-americana do delsartismo foi até mesmo levada de volta à Europa com vistas à educação física e novas práticas de dança na Alemanha e outros países. Entrementes, como discutido anteriormente, além de promover a causa da cultura física e da expressão das mulheres, alguns proponentes também promoviam princípios estéticos para defender a artistagem na vida cotidiana - no vestuário, decoração doméstica, interações sociais e qualquer outra coisa que poderia ser melhorada desse modo. Foi precisamente nesse aspecto do delsartismo americano que alguns críticos, como Shawn, viram uma distorção das intenções do mestre - "[...] uma inversão e falsificação da ciência que Del- 
sarte ensinou" (1974, p. 11). No entanto, sua delsarteana mais reverenciada, Henrietta Hovey, havia sido uma das líderes na popularização do delsartismo americano em todos esses aspectos.

Enquanto alguns dos delsarteanos norte-americanos, como Genevieve Stebbins, dançavam e ensinavam dança ou formas semelhantes como parte de seu trabalho delsarteano no final do século XIX, a influência do delsartismo no desenvolvimento da dança do século XX começou realmente com o trabalho de Shawn, St. Denis e sua companhia, a Denishawn. O conhecimento e experiência de Shawn e St. Denis com o sistema Delsarte havia passado por diversas fases antes de seu contato com Hovey. Em 1915, no entanto, Shawn embarcou em uma experiência mais concreta e imediata com o delsartismo do que ele havia conhecido anteriormente, e, é claro, isso era compartilhado com St. Denis e com os membros da companhia e da escola. Shawn teve aulas particulares com Hovey nos verões de 1915, 1916 e 1917, e também a convidou para dar palestras sobre delsartismo na Denishawn. Ela se tornou a mais importante fonte de conhecimento delsarteano para Shawn e provavelmente inspirou a sua pesquisa intensiva sobre o amplo espectro da literatura delsarteana, o qual resultou no livro Every Little Movement. Penso que o delsartismo que Shawn veio a conhecer ao longo dos anos serviu como uma orientação poderosa, contínua e crucial para o seu desenvolvimento como artista, professor, teórico, escritor e líder de dança nos Estados Unidos - influenciando significativamente seus estudantes e seguidores.

Apesar do próprio François Delsarte nunca ter demonstrado o mínimo interesse pela dança, seus conceitos e manifestações teóricas e práticas têm sido seminais no desenvolvimento do gênero de concerto do século XX, a dança moderna. Desde a publicação de Shawn, Every Little Movement, a contribuição da teoria e prática delsarteanas para a história da dança do século XX tem sido reconhecida por historiadores e estudiosos - em alguns brevemente, em outros com discussões e análises mais amplas. O livro de Shawn oferece um estudo cuidadosamente pesquisado e bem escrito sobre o sistema de Delsarte e sua relação com a dança, como também uma discussão sobre 
a literatura mais relevante publicada até a década de 1960 . Ele é uma das fontes mais confiáveis e significativas sobre o assunto em língua inglesa.

Os princípios fundamentais do delsartismo, conforme estabelecidos por Delsarte, foram retransmitidos por quem havia estudado com o próprio mestre e, então, por seus alunos e alunos de seus alunos - retransmitidos tanto através de instrução pessoal quanto por escritos sobre o sistema -, alguns publicaram na Europa, porém, a maior parte nos Estados Unidos. Com uma história que se desenvolveu ao longo de várias gerações de seguidores e entre os vários ramos de atuação, oratória, canto, cultura, expressão física e dança, não é nenhuma surpresa que diferentes ênfases e adaptações tenham surgido no âmbito do sistema Delsarte, além de uma gama de interpretações, usos e métodos de ensino. É claro que essas adaptações e a proliferação do delsartismo nos Estados Unidos levaram a disputas sobre o que era autêntico e o que era um falseamento. Embora seja compreensível que os antigos seguidores profissionais de Delsarte tenham se sentido obrigados a defender quaisquer aspectos do complexo que cada um via como o verdadeiro Delsarte, de maneira a repudiar aquilo que consideravam falso, penso ser mais útil considerar os vários aspectos do desenvolvimento histórico da teoria e prática de Delsarte sem nenhum preconceito, apreciando as diversas maneiras com que elas têm funcionado e servido a várias necessidades.

Nas últimas décadas, o interesse pelo trabalho de Delsarte vem crescendo na Europa e nos Estados Unidos. Tem havido publicações, conferências e exposições na França e na Itália; patrocínio do Centre National de la Danse em Paris para uma tradução para o francês da obra de Shawn, Every Little Movement; além de uma edição do Mime Journal ${ }^{6}$ dedicada à pesquisa delsarteana. A evidência mais recente da importância duradoura do trabalho de Delsarte e de seus seguidores foram os eventos que ocorreram em 2011, em Stuttgart, Paris e Pádua, em comemoração aos 200 anos de nascimento de Delsarte e para difundir ainda mais as pesquisas e práticas dos vários aspectos do delsartismo. 


\section{Notas}

${ }^{1}$ Para obter informações sobre elocução no século XIX, ver os periódicos de Wallace; Curry; e Edgar S. Werner, que foram publicados com três títulos diferentes: The Voice (1879-1888), Werner's Voice Magazine (1889-1892) e Werner's Magazine (1893-1902).

${ }^{2}$ As informações biográficas de Mackaye vêm de Percy Mackaye; Mrs. Steele Mackaye; McTEague; e da Mackaye Collection na Datmouth College Library.

${ }^{3}$ Informações biográficas de diferentes graus de confiabilidade podem ser encontradas em Willbor; Shelton; Greenlee e Greenlee; "Delsartism in America"; publicações da New York School of Expression; e notícias ou artigos nas revistas Werner ou outros periódicos.

${ }^{4}$ Para mais sobre a teoria e prática de Stebbins, ver Stebbins 1888, 1893, 1895, 1902, 1913.

${ }^{5}$ Para informações biográficas sobre Hovey, ver Macdonald; Meckel; Shawn; Who's Who in America (de 1899-1900 até mais de 20 anos após a morte de Hovey em 1918); materiais nos papéis de Richard Hovery na Dartmouth College Library; e artigos e notícias nas revistas Werner e outros periódicos.

${ }^{6}$ N. T.: Mime Journal é um periódico publicado anualmente pelo Pomona College, de Claremont, EUA, tendo como editor, Thomas Leabhart. 


\section{Referências}

CURRY, Samuel S. The Province of Expression; A Search for Principles Underlying Adequate Methods of Developing Dramatic and Oratoric Delivery. Boston: School of Expression, 1891.

DElaumosne, Abbé. Pratique de L'art Oratoire de Delsarte. Tradução para o inglês de Frances A. Shaw: "The Delsarte System”, Delsarte System of Oratory. 1. ed. (New York: Edgar S. Werner, 1882), 1874.

DELSARTISM in America. Werner's Voice Magazine, v. 14, p. 59-64, mar. 1892.

GREENLEE, Ralph Stebbins; GREENLEE, Robert Lemuel. The Stebbins Genealogy. Chicago: McDonohue and Co, 1904.

MACDONALD, Allan Houston. Richard Hovey: man and craftsman. Durham: Duke University Press, 1957.

MACKAYE, Mrs. Steele (Mary Medberry). Steele Mackaye and François Delsarte. Werner's Voice Magazine, v. 14, jul. 1892.

MACKAYE, Percy. Epoch: The Life of Steele Mackaye, Genius of the Theatre in Relation to His Times and Contemporaries. 2 volumes. New York: Boni and Liveright, 1927.

MCTEAGUE, James H. Before Stanislavsky: american professional acting schools and acting theory, 1875-1925. Metuchen: Scarecrow Press, 1993.

MECKEL, Richard A. Henrietta Russell: Delsartean Prophet to the Gilded Age. Journal of American Culture, v. 12, n. 1, p. 65-78, 1989.

NEW YORK SCHOOL of Expression. 1893 prospectus; 1910-11 catalogue. (ambos na Theatre Collection of the New York Public Library Performing Arts Collections)

RUYTER, Nancy Lee Chalfa; LEABHART, Thomas (Org.). ESSAYS on François Delsarte. Mime Journal, 2004-2005. Claremont, CA: Pomona College Theatre Department.

RUYTER, Nancy Lee Chalfa. American Delsartism: precursor of an American Dance Art. Educational Theatre Journal, v. 25, p. 421-435, dez. 1973.

RUYTER, Nancy Lee Chalfa. Reformers and Visionaries: the americanization of the art of dance. New York: Dance Horizons, 1979.

RUYTER, Nancy Lee Chalfa. The Intellectual World of Genevieve Stebbins. Dance Chronicle, London, v. 11, n. 3, p. 389-397, 1988.

RUYTER, Nancy Lee Chalfa. Antique Longings: Genevieve Stebbins and American Delsartean Performance. In: FOSTER, Susan Leigh (Org.). Corporealities: Dancing, Knowledge, Culture and Power. London: Routledge, 1996. P. 70-89.

RUYTER, Nancy Lee Chalfa. The Delsarte Heritage. Dance Research, Edinburgh, v. 14, n. 1, p. 62-74, abr. 1996.

RUYTER, Nancy Lee Chalfa. The Cultivation of Body and Mind in Nineteenth-Century American Delsartism. Westport, CT: Greenwood Press, 1999. 
SHAWN, Ted. Every Little Movement: A Book about François Delsarte. Republication of $2^{\text {nd }}$ rev. \& enl. ed. de 1963. New York: Dance Horizons, 1974.

SHELTON, Suzanne. The Influence of Genevieve Stebbins on the Early Career of Ruth St. Denis. In: WOODRUFF, Dianne L. (Org.). Essays in Dance Research from the Fifth CORD Conference, Philadelphia, nov. 1976. Dance Research Annual IX. New York: Congress on Research in Dance, 1978. P. 33-49.

STEBBINS, Genevieve. Society Gymnastics and Voice Culture, Adapted from the Delsarte System. New York: Edgar S. Werner, 1888.

STEBBINS, Genevieve. Dynamic Breathing and Harmonic Gymnastics. New York: Edgar S. Werner, 1893.

STEBBINS, Genevieve. Genevieve Stebbins Drills. New York: Edgar S. Werner, 1895.

STEBBINS, Genevieve. Delsarte System of Expression. 6a. ed. New York: Edgar S Werner, 1902. Reedição New York: Dance Horizons, 1977. (Primeira edição 1885. Edições anteriores foram intituladas Delsarte System of Dramatic Expression. Materiais novos foram adicionados em edições subsequentes; a mais completa, portanto, é a $6^{\text {a }}$ )

STEBBINS, Genevieve. The Genevieve Stebbins System of Physical Training. enl. ed. New York: Edgar S. Werner, 1913. (Primeira edição 1898)

WALLACE, Karl R. (Org.). History of Speech Education in America. New York: AppletonCentury-Crofts, 1954.

WILBOR, Elsie M. (Org.). Werner's Directory of Elocutionists, Readers, Lecturers, and Other Public Instructors and Entertainers. New York: Edgar S. Werner, 1887.

ZORN, John W. (Org.). The Essential Delsarte. Metuchen, NJ: Scarecrow Press, 1968.

Nancy Lee Chalfa Ruyter é formada em Artes e doutora em História. Ela é historiadora da dança, coreógrafa e professora. Professora de Dança na Universidade da Califórnia, Irvine, onde leciona desde 1982. Na área de dança, possui treinamento em balé, dança moderna e várias formas de danças típicas. Suas publicações incluem Reformers and Visionaries: The Americanization of the Art of Dance (1979); The Cultivation of Body and Mind in 19th-Century American Delsartism (1999); e muitos outros sobre o sistema Delsarte e seus usos, dança espanhola, dança dos Bálcãs, teatro latino-americano e movimento teatral.

E-mail: nlruyter@uci.edu

Traduzido do original em inglês por Martin Dahlström Heuser e revisado por Gilberto Icle. 